\title{
Signal Processing in Cognitive Radio
}

\author{
To share frequencies without interfering, cognitive radio systems need to \\ constantly monitor for the presence of licensed users and to continuously \\ adjust the spectrum of their transmitted signal.
}

By Jun Ma, Geoffrey Ye Li, Fellow IEeE, and Biing Hwang (Fred) Juang, Fellow IEeE

\begin{abstract}
Cognitive radio allows for usage of licensed frequency bands by unlicensed users. However, these unlicensed (cognitive) users need to monitor the spectrum continuously to avoid possible interference with the licensed (primary) users. Apart from this, cognitive radio is expected to learn from its surroundings and perform functions that best serve its users. Such an adaptive technology naturally presents unique signal-processing challenges. In this paper, we describe the fundamental signal-processing aspects involved in developing a fully functional cognitive radio network, including spectrum sensing and spectrum sculpting.
\end{abstract}

KEYWORDS | Cyclostationary detection; energy detection; matched filter detection; spectrum sculpting; spectrum sensing

\section{INTRODUCTION}

The recent rapid growth of wireless communications has made the problem of spectrum utilization ever more critical. On one hand, the increasing diversity (voice, short message, Web, and multimedia) and demand of high quality-of-service (QoS) applications have resulted in overcrowding of the allocated (officially sanctioned) spectrum bands, leading to significantly reduced levels of user satisfaction. The problem is particularly serious in communication-intensive situations such as after a ballgame or in a massive emergency (e.g., the 9/11 attacks). On the other hand, major licensed bands, such as those allocated for television broadcasting, amateur radio, and

Manuscript received November 5, 2008. First published April 24, 2009; current version published May 1, 2009. This work was supported in part by the National Science Foundation under Grant 0721580 and Huawei Technologies Co., Ltd., under a Research Gift.

The authors are with School of Electrical and Computer Engineering, Georgia Institute of Technology, Atlanta, GA 30308 uSA (e-mail: biing.juang@ece.gatech.edu; liye@ece.gatech.edu; junma@ece.gatech.edu).

Digital Object Identifier: 10.1109/JPROC.2009.2015707 paging, have been found to be grossly underutilized, resulting in spectrum wastage. For example, recent studies by the Federal Communications Commission (FCC) show that the spectrum utilization in the $0-6 \mathrm{GHz}$ band varies from $15 \%$ to $85 \%$ [1]. This has prompted the FCC to propose the opening of licensed bands to unlicensed users and given birth to cognitive radio [2]. The IEEE has formed a working group (IEEE 802.22) to develop an air interface for opportunistic secondary access to the TV spectrum via the cognitive radio technology. The guiding philosophy of cognitive radio is to allow universal maximization of the spectrum utilization insofar as the unlicensed users do not cause degradation of service upon the original license holders. In practice, the unlicensed users, also called the cognitive users, need to monitor the spectrum activities continuously to find a suitable spectrum band for possible utilization and to avoid possible interference to the licensed users, also called the primary users. Since the primary users have the priority of service, the above spectrum sensing by cognitive users includes detection of possible collision when a primary user becomes active in the spectrum momentarily occupied by a cognitive user and relocation of the communication channels. Given geographical constraints, how accurately can the cognitive users detect the presence of the licensed user? Also, how should the cognitive users prioritize the potential bands for utilization so as to minimize the need of channel relocation, or to maximize the usage time between channel relocation, in response to potential positive detection of a primary user activity? There are many challenges that need to be resolved before a fully functional cognitive radio network can be implemented.

In fact, any cognitive radio ( $\mathrm{CR}$ ) network that can be deployed in practice needs to have the following minimal features.

- A unified cross-layer cognitive network architecture equipped to handle diverse QoS requirements. 
- Efficient spectrum sensing techniques that provide continuous monitoring of the presence of multicarriers in the CR network.

- Dynamic spectrum access methods that adapt to the fluctuating nature of the CR network and allocate bandwidth accordingly.

- Adaptive spectrum sculpting at the transmitter end that causes minimal or no interference to the primary users occupying adjacent bands.

To build a highly adaptive radio technology that learns from the environment to best serve its users, novel signaprocessing techniques that are channel-aware and cognitive need to be developed. In [3], fundamental issues specific to cognitive radio, including radio-scene analysis, channel-state estimation and predictive modeling, transmitpower control, and dynamic spectrum management, have been first investigated, presenting a big picture of cognitive radio. In [4], signal-processing issues in the context of spectrum sensing implementation in CR networks have been investigated. In this paper, we focus on the signal processing aspects demanded of each of the above-mentioned features in CR networks. More precisely, we investigate spectrum sensing and spectrum sculpting in the context of cognitive radio.

The rest of this paper is organized as follows. In Section II, we formulate the problem of spectrum sensing in CR networks and describe the basic techniques that may be employed for detection of the primary signal. These techniques require a different amount of knowledge of the primary signal characteristics and are applicable to different scenarios. In order that the primary signal is detected as quickly as possible, it is sometimes necessary to employ more than one cognitive detector for cooperative spectrum sensing. In Section III, we describe cooperative detection techniques to boost the overall detection capability of a CR network. In Section IV, we discuss the problem of adaptive spectrum sculpting that is a necessary functionality in operational CR networks. In particular, we investigate the multicarrier techniques and transformdomain communication system (TDCS) that can be used in the physical layer to accomplish this objective. We conclude this paper with a summary in Section V.

\section{SPECTRUM SENSING: BASIC TECHNIQUES}

As secondary users, CR operators are allowed to utilize a licensed band only when they do not cause interference to the primary users. Spectrum sensing aims at monitoring the usage and characteristics of the covered spectral band(s) and is thus required by $\mathrm{CR}$ users both before and during the use of licensed spectrum bands. In this section, we first formulate the problem of spectrum sensing in $\mathrm{CR}$ and then describe the basic spectrum sensing techniques, including energy detection, cyclostationary detection, pilot-based coherent detection, and some other detection techniques.

\section{A. Spectrum Holes}

$\mathrm{CR}$ is designed to identify and scavenge the spectrum holes in the licensed spectrum bands. A spectrum hole is defined as a licensed spectrum band that can be used by CR users without interfering the primary or licensed users. Generally spectrum holes can be broadly divided into two categories: temporal spectrum holes and spatial spectrum holes, which are shown in Fig. 1(a) and 1(b), respectively.

A temporal spectrum hole means that there is no primary transmission over the spectrum band of interest during the time of sensing (over a reasonable period); hence, this band can be utilized by CR users in the current time slot. For the temporal spectrum holes, as indicated in Fig. 1(a), the secondary users are located in the coverage area of the primary transmission. Consequently, it is relatively easy to detect the presence or absence of the primary user activity since CR users only need to have a similar detection sensitivity as regular primary receivers and, more importantly, identifying the presence of a primary signal is much easier than demodulating and decoding it. Therefore, spectrum sensing in this case does not pose a onerous demand on signal processing.

A spatial spectrum hole exists when the spectrum band of interest is occupied by the primary transmission only in a restricted area; hence, this band can be utilized by $\mathrm{CR}$ users well outside this area [5]. ${ }^{1}$ In contrast with the utilization of temporal spectrum holes, secondary users utilizing spatial spectrum holes work outside the coverage of the primary transmission, as indicated in Fig. 1(b). Since there are no primary receivers outside the coverage area, secondary communication over the licensed band is allowed if only the secondary transmitter does not interfere with the primary transmission and reception within the coverage area. To accomplish this, the secondary transmitter has to successfully detect the presence of the primary signal at any location where the secondary transmission may cause intolerable interference to the possible nearby primary receiver. Since the secondary users fall outside the coverage area of the primary transmission, detection of the primary signal in this case is a challenging task.

Here we elaborate the stringent signal processing requirements for detection of spatial spectrum holes from a geographic perspective as in [6] and [7]. Denote $P_{p}$ and $P_{s}$ as the transmit powers of the primary and the secondary transmitters, respectively, $P_{n}$ as the noise power at the primary and the secondary receivers, $R$ as the maximum distance between the primary transmitter and the coverage edge, and $D$ as the minimum distance between the secondary transmitter and the coverage edge. Further define $\Gamma_{\min }$ as the lowest signal-to-noise ratio (SNR) level at the primary receiver that guarantees successful primary signal reception. In order for the

${ }^{1}$ In theory, the area is defined not necessarily in the geographical sense but in the communication sense; a covered area or coverage is where the link between a receiver and the target transmitter is sustainable. 


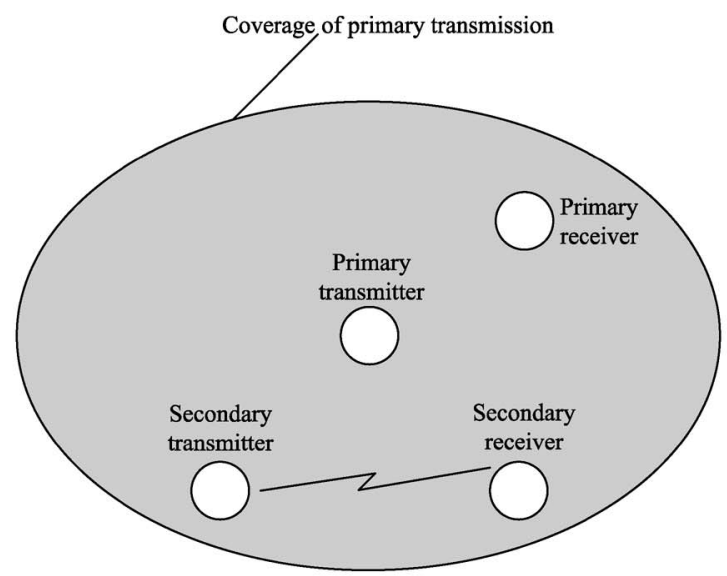

(a)

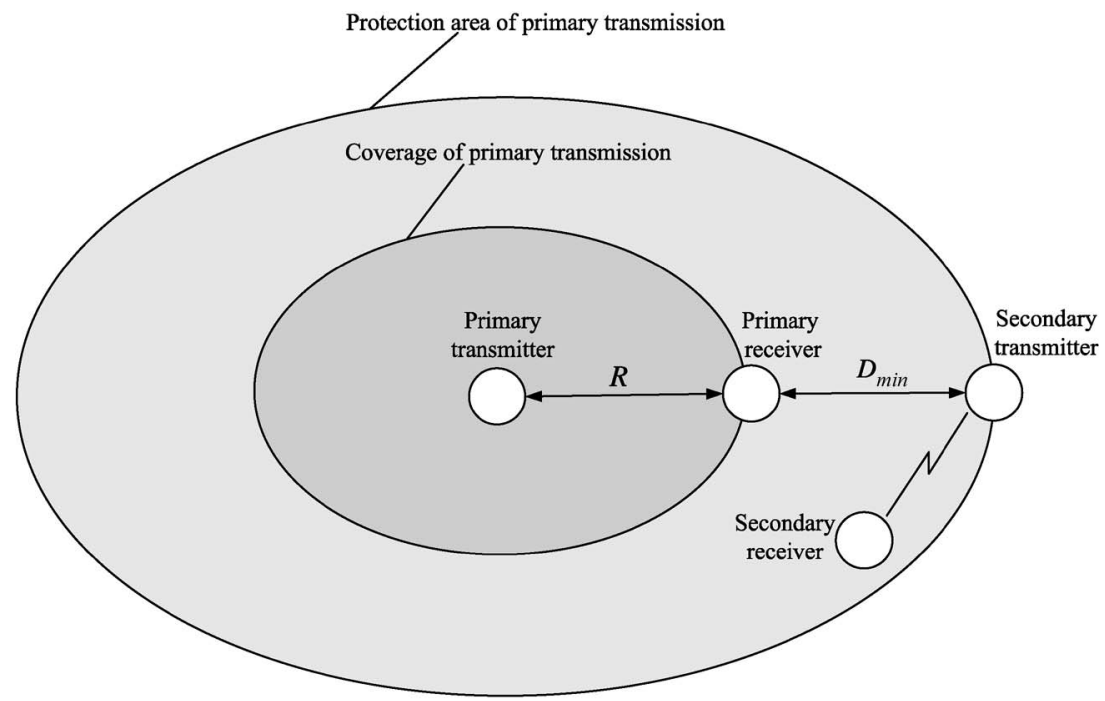

(b)

Fig. 1. Spectrum holes for secondary communication. (a) Temporal spectrum holes and (b) spatial spectrum holes.

primary receivers located the furthest from the primary transmitter to be able to detect the primary signal, we must have

$$
\frac{P_{p} L(R)}{P_{n}} \geq \Gamma_{\min }
$$

where $L(\cdot)$ denotes the power loss for a given distance, including the path loss, shadowing, and multipath fading. Define

$$
\delta=\frac{P_{p} L(R)}{P_{n} \Gamma_{\min }}
$$

which is the power margin factor of the primary system.
Under the worst case that the primary receiver lies on the coverage edge, the furthest $(R)$ from the primary transmitter, and the nearest $(D)$ from the secondary transmitter, as indicated in Fig. 1(b), the received SNR at the primary receiver is given by

$$
\gamma=\frac{P_{p} L(R)}{P_{s} L(D)+P_{n}} .
$$

For $\gamma$ to be above $\Gamma_{\min }$, it is required that

$$
\begin{aligned}
D \geq D_{\min } & =L^{-1}\left(\frac{P_{p} L(R)}{P_{s} \Gamma_{\min }}-\frac{P_{n}}{P_{s}}\right) \\
& =L^{-1}\left((\delta-1) \frac{P_{n}}{P_{s}}\right)
\end{aligned}
$$


where $L^{-1}(\cdot)$ denotes the inverse function of $L(\cdot)$. According to (4), $D_{\min }$ increases with the transmit power of the secondary transmitter $P_{s}$ and decreases with the power margin factor of the primary system $\delta$. Equation (4) also indicates that, to avoid intolerable interference with the primary transmission, any secondary transmitter with transmit power $P_{s}$ must successfully detect the presence of primary signal when it is $R+D_{\min }$ away from the primary transmitter. In other words, there exists a protection area for the primary transmission in which the presence of primary signal must be successfully detected by secondary transmitters to avoid interfering with the primary transmission. As indicated in Fig. 1(b), the protection area of primary transmission contains and is larger than the primary transmission coverage. Since the secondary users are required to detect the presence of primary signal well outside the primary transmission coverage, the detection of spatial spectrum holes entails advanced spectrum sensing techniques. Generally, secondary users utilizing spatial spectrum holes must have a much higher detection sensitivity than regular primary receivers.

\section{B. Primary Signal Detection}

Whether for the detection of temporal or spatial spectrum holes, spectrum sensing in CR involves deciding whether the primary signal is present or not from the observed signals. It can be formulated as the following two hypotheses:

$$
y(t)= \begin{cases}i(t)+w(t), & \mathcal{H}_{0} \\ s(t)+i(t)+w(t), & \mathcal{H}_{1}\end{cases}
$$

where $y(t)$ is the received signal at the CR user, $s(t)$ is the primary signal, $i(t)$ is interference, ${ }^{2}$ and $w(t)$ is the additive white Gaussian noise (AWGN). In (5), $\mathcal{H}_{0}$ and $\mathcal{H}_{1}$ denote the hypotheses corresponding to the absence and presence of the primary signal, respectively. Thus from the observation $y(t)$, the CR user needs to decide between $\mathcal{H}_{0}$ and $\mathcal{H}_{1}$.

For different licensed band(s), primary signals have different characteristics. The 802.22 wireless regional-area network (WRAN) is developed to work in licensed TV bands; therefore the primary signal is the Advanced Television Systems Committee (ATSC) digital TV signal or the wireless microphone signal. For CR networks to utilize the temporally idle spectrum bands allocated to the $3 \mathrm{G}$ cellular mobile communication system, the primary signal may be direct spread code-division multiple access (DS-CDMA) signal or orthogonal frequency-division multiplexing (OFDM) signal for its long-term evaluation (LTE) version. In this paper, we do not restrict the primary signal to any waveform. Instead, we exploit the characteristics of the

\footnotetext{
${ }^{2}$ The difference between interference and noise is that interference is undesired man-made colored signal while noise is white and statistically Gaussian. When interference from various sources in the environment approximates Gaussian and white, it is regarded as noise.
}

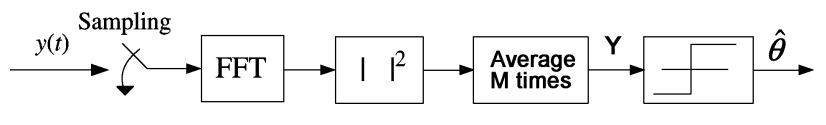

Fig. 2. Schematic representation of the energy detector over a spectrum subband of interest.

primary signal that are generally known to the public for spectrum sensing. Up to now, various spectrum sensing techniques have been proposed to utilize the characteristics or the a priori knowledge of the primary signal. In the rest of this section, we will focus on the basic spectrum sensing techniques that can be implemented at an individual CR user. Cooperative spectrum sensing techniques will be addressed in the next section.

\section{Energy Detection}

Energy detection [8] is the simplest spectrum sensing technique, which is shown in Fig. 2. An energy detector (ED) simply treats the primary signal as noise and decides on the presence or absence of the primary signal based on the energy of the observed signal. Since it does not need any a priori knowledge of the primary signal, the ED is robust to the variation of the primary signal. Moreover, the ED does not involve complicated signal processing and has low complexity.

In practice, energy detection is especially suitable for wide-band spectrum sensing. In this case, the simultaneous sensing of a number of subbands can be realized by simply scanning the power spectral density (PSD) of the received wide-band signal. In practice, it is advisable to complete wide-band spectrum sensing via two stages. In the first stage, low-complexity energy detection is applied to search for possible idle subbands; in the second stage, more advanced spectrum sensing techniques with a higher detection sensitivity and thereby higher complexity, such as cyclostationary detection, are applied to the subband candidates to determine whether they are actually available for secondary usage.

Performance Analysis: As indicated in Fig. 2, the spectral component on each spectrum subband of interest is obtained from the fast Fourier transform (FFT) of the sampled received signal. Then the test statistics of the ED is obtained as the observed energy summation within $M$ consecutive segments, i.e., ${ }^{3}$

$$
Y= \begin{cases}\sum_{m=1}^{M}|W(m)|^{2}, & \mathcal{H}_{0} \\ \sum_{m=1}^{M}|S(m)+W(m)|^{2}, & \mathcal{H}_{1}\end{cases}
$$

where $S(m)$ and $W(m)$ denote the spectral components of the received primary signal and the white noise on the subband

\footnotetext{
${ }^{3}$ To facilitate analysis, here we ignore the interference component in the received signal.
} 
of interest in the $m$ th segment, respectively. The decision of the ED regarding the subband of interest is given by

$$
\hat{\theta}= \begin{cases}\mathcal{H}_{1}, & \text { if } Y>\lambda \\ \mathcal{H}_{0}, & \text { if } Y<\lambda\end{cases}
$$

where the threshold $\lambda$ is chosen to satisfy a target false-alarm probability. $^{4}$

Without loss of generality, we assume the noise $W(m)$ is white complex Gaussian with zero mean and variance two. Define the instantaneous SNR of the received primary signal within the current $M$ segments as

$$
\gamma=\frac{1}{2 M} \sum_{m=1}^{M}|S(m)|^{2} .
$$

Then the test statistics of the ED $Y$ follows a central chisquare distribution with $2 M$ degrees of freedom under $\mathcal{H}_{0}$, and a noncentral chi-square distribution with $2 M$ degrees of freedom and a noncentrality parameter $\mu=\sum_{m=1}^{M} \mid$ $\left.S(m)\right|^{2}=2 M \gamma$ under $\mathcal{H}_{1}$, i.e.,

$$
f_{Y}(Y) \sim \begin{cases}\chi_{2 M}^{2}, & \mathcal{H}_{0} \\ \chi_{2 M}^{2}(\mu), & \mathcal{H}_{1}\end{cases}
$$

where $f_{Y}(Y)$ denotes the probability density function (pdf) of $Y$ and $\chi_{M}^{2}$ and $\chi_{M}^{2}(\mu)$ denote a central and noncentral chi-square distribution, respectively. Thus the false-alarm probability $P_{F}=P\left(Y>\lambda \mid \mathcal{H}_{0}\right)$ can be expressed as [9]

$$
P_{F}=\frac{\Gamma\left(M, \frac{\lambda}{2}\right)}{\Gamma(M)}
$$

where $\Gamma(\cdot)$ and $\Gamma(\cdot, \cdot)$ denote the gamma function and the upper incomplete gamma function [10], respectively. Given the target false-alarm probability, the threshold $\lambda$ can be uniquely determined based on (10). Once $\lambda$ is determined, the detection probability $P_{D}=P\left(Y>\lambda \mid \mathcal{H}_{1}\right)$ can be obtained by [9]

$$
\begin{aligned}
P_{D} & =\int_{0}^{+\infty} P\left(Y>\lambda \mid \mathcal{H}_{1}, \mu\right) f_{\mu}(\mu) d \mu \\
& =\int_{0}^{+\infty} Q_{M}(\sqrt{\mu}, \sqrt{\lambda}) f_{\mu}(\mu) d \mu
\end{aligned}
$$

\footnotetext{
${ }^{4}$ The threshold defines an operating point on the "receiver operating curve" over two performance parameters - the false-alarm probability and the missed detection probability. Here, the false-alarm probability is customarily chosen as the primary operating specification.
}

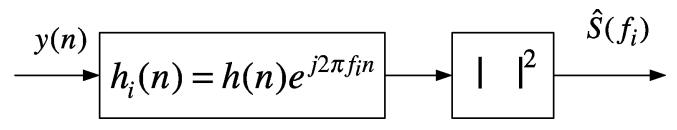

Fig. 3. Principle of filter bank power spectrum estimation.

where $Q_{M}(\cdot, \cdot)$ is the generalized Marcum $Q$-function and $f_{\mu}(\mu)$ is the pdf of $\mu$. In [9], a closed-form expression of the detection probability of the ED over general Nakagami fading channel has been derived.

Advanced Power Spectrum Estimation Techniques: As indicated in Fig. 2, energy-detection-based wide-band spectrum sensing can monitor multiple subbands simultaneously by scanning the estimated power spectrum of the received wide-band signal. ${ }^{5}$ Obviously, an accurate power spectrum estimate is vital to successful detection of idle subbands. Over a wide spectrum band of interest, some subbands may be occupied by licensed services with significantly different transmit powers and the others may be unoccupied and filled with random noise only. As a result, the power spectrum estimator must have a high spectral dynamic range (SDR), which is defined as the ratio of the maximum and the minimum spectral powers that are distinguishable by this estimator [11]. The ED shown in Fig. 2 applies the simplest periodogram spectral estimator [12]. In the following, we will investigate more advanced nonparametric power spectrum estimation techniques that can be applied to improve the performance of energy detection.

Here we refer to [11] and investigate power spectral estimation from the perspective of filter bank. The principle of filter bank power spectrum estimation is shown in Fig. 3. Suppose there are altogether $N$ subbands in the whole spectrum band of interest. As indicated in the figure, the ith $(0 \leq i \leq N-1)$ subfilter of the filter bank $h_{i}(n)=h(n) e^{j 2 \pi f_{i} n}$ is utilized to extract the spectral component of the received signal over the ith subband with the normalized center frequency $f_{i}=i / N$, where $h(n)$, the low-pass filter used to realize the zeroth subband, is called the prototype filter of the filter bank. Obviously, the selection of the prototype filter determines the accuracy of spectral estimation. More specifically, the magnitude of the side lobes of the prototype filter determines the amount of power leakage from the neighboring subbands to the subband of interest and, hence, determines the SDR of the power spectrum estimator. Therefore, in order to improve the performance of power spectrum estimation, the side lobes of the prototype filter should be reduced.

\footnotetext{
${ }^{5}$ Generally speaking, the received stochastic signal at the CR user is nonstationary, i.e., its statistics varies with time. In practice, the received signal is usually sectioned and analyzed burst by burst, with each burst short enough to ensure pseudostationary and yet long enough to produce an accurate spectral estimate [3].
} 
For the classical periodogram spectral estimator, the signal energy on the ith subband is estimated by

$$
\begin{aligned}
\hat{S}\left(f_{i}\right) & =\left|\sum_{n=0}^{N-1} y(n) w(n) e^{-j 2 \pi f_{i} n}\right|^{2} \\
& =\left|\sum_{n=0}^{N-1} w(n) e^{j 2 \pi f_{i} n} y(N-1-n)\right|^{2}
\end{aligned}
$$

where $w(n)$ denotes a symmetric window function, i.e., $w(n)=w(N-1-n), 0 \leq n \leq N-1$. Equation (12) indicates that the periodogram spectral estimator is actually a filter-bank-based one with the prototype filter being the window function $w(n)$. For the simple FFT-based power spectrum estimator in Fig. 2, a rectangular window is implied, which, as the prototype filter, has large side lobes and therefore inevitably results in a limited SDR of the estimator.

To improve the performance of periodogram spectral estimator, various window functions with small side lobes have been proposed [13] to preprocess the received signal before FFT operation, and this is called tapering in the literature. Though tapering effectively reduces the bias of the power spectrum estimate of a stochastic signal, it unfortunately increases its variance because of the information loss caused by the truncation of the time-domain windowing. To mitigate such information loss, it has been proposed to utilize multiple tapers, or prototype filters, in power spectrum estimation so as to reduce the variance of the estimate. This is called multitaper spectral estimation [14]. In the multitaper method, a special family of sequences known as the Slepian sequences are usually applied as the tapers for spectral estimation. The Slepian sequences have two basic characteristics: first, their Fourier transforms have the maximal energy concentration in the main lobe, which means the least power leakage when applied for spectral estimation; secondly, they are orthogonal to each other, which means that the estimate outputs of different estimation entities employing different tapers are uncorrelated if only the variation of the signal spectrum over each subband is negligible. As a result, averaging these estimates will result in a minimum variance. Due to the two characteristics of the Slepian sequences, the multitaper spectral estimation has been shown nearly optimal in the sense that it almost achieves the Cramer-Rao bound for a nonparametric spectral estimator [15]. Therefore, the multitaper spectral estimation has been recommended by Haykin in [3] as a promising power spectrum estimation technique for energy-detection-based wide-band spectrum sensing.

Although the multitaper spectral estimation has nearly optimal performance, it involves high implementation complexity. Recently, filter bank multicarrier communication techniques, including the OFDM offset quadrature amplitude modulation (OQAM), cosine modulated multitone (CMT), and filtered multitone (FMT), have been proposed for the physical layer of CR systems [16]. These filter bank multicarrier techniques provide a high flexibility in adapting the spectrum shape of the transmitted signal in accordance with the available licensed bands and, at the same time, can better mitigate the mutual interference between the primary and secondary users than the conventional OFDM technique, as will be discussed in detail in Section IV. Since the prototype filters of these filter bank multicarrier techniques have small side lobes, these filter banks can be utilized for accurate power spectral estimation in wide-band spectrum sensing. It has been shown in [11] that such filter bank power spectrum estimators can achieve a similar SDR as the multitaper method with a lower complexity. More importantly, since the filter bank has already been exploited as the physical layer of $\mathrm{CR}$ systems for multicarrier communication, it can be utilized for spectrum sensing without any additional cost.

Limitations of Energy Detection: So far, we have assumed that the noise power is exactly known for the ED. However, this assumption may be invalid under certain environments. The noise usually consists of the local thermal noise and the environment noise. In practice, the local thermal noise changes over time because of temperature variations at the receiver; the environment noise, which is an aggregation of random signals from various sources in the environment, also varies with time. As a result, though the central limit theorem is invoked to justify the Gaussian nature of noise, it is practically impossible to know the current noise power exactly. Moreover, the existence of variable in-band interference makes the situation even worse. As a result, such noise and interference power uncertainty severely degrades the performance of energy detection.

Denote $\sigma_{n}^{2}$ and $\sigma_{e}^{2}$ to be the actual noise and interference power and its estimate, respectively. Suppose there is an $x \mathrm{~dB}$ uncertainty in the noise and interference power estimation. Then $\sigma_{e}^{2}$ may take any value between $\sigma_{n}^{2} 10^{-(x / 10)}$ and $\sigma_{n}^{2} 10^{x / 10}$, i.e., $\sigma_{e}^{2} \in\left[10^{-(x / 10)} \sigma_{n}^{2}, 10^{x / 10} \sigma_{n}^{2}\right]$. Therefore, the primary signal can be always detected only when the power of the received signal is greater than the threshold $\sigma_{T}^{2}=10^{x / 10} \sigma_{e}^{2}$. Under the worst case that $\sigma_{e}^{2}=10^{x / 10} \sigma_{n}^{2}$, $\sigma_{T}^{2}=10^{2 x / 10} \sigma_{n}^{2}$, and, as a result, the ED will fail to detect the presence of the primary signal if the power of the received primary signal is smaller than $\left(\sigma_{T}^{2}-\sigma_{n}^{2}\right)$. That is, there is an SNR wall for the ED given by

$$
\gamma_{w}=\frac{\sigma_{T}^{2}-\sigma_{n}^{2}}{\sigma_{n}^{2}}=10^{\frac{2 x}{10}}-1 .
$$

In other words, for all received SNRs smaller than $\gamma_{w}$, there exists a possibility that the ED is not able to distinguish between the two hypotheses $\mathcal{H}_{0}$ and $\mathcal{H}_{1}$, no matter how many samples the ED utilizes. In [17], bounds on the SNR wall of general detectors based on the $2 k$ th moments have been presented for a slightly more general noise uncertainty model. 
Even if the actual noise and interference power is known exactly, the performance of energy detection is still limited by its inability to differentiate the primary signal from the interference and noise, especially when it comes to low-power primary signals such as spread spectrum signals. While the ED is a good option when the CR user knows nothing about the primary signal or when complexity is the main concern, more complicated and accurate spectrum sensing techniques that exploit the primary signal characteristics should be employed to achieve a better detection performance. This is particularly meaningful in CR networks since the primary signal has certain signatures, such as modulation, coding, and pilot symbols, that can be extracted to improve the detection capability. In the rest of this section, we will investigate various spectrum sensing techniques that utilize different characteristics of the primary signal and are able to differentiate the primary signal from the interference and noise.

\section{Cyclostationary Detection}

Man-made signals are generally nonstationary. Some of them are cyclostationary, i.e., their statistics exhibit periodicity, which may be caused by modulation and coding or even be intentionally produced to aid channel estimation and synchronization. Such periodicity can be utilized for detection of a random signal with a particular modulation type in a background of noise and other modulated signals. This is called cyclostationary detection. Mathematically, cyclostationary detection is realized by analyzing the cyclic autocorrelation function (CAF) [18] of the received signal, or, equivalently, its two-dimensional spectrum correlation function (SCF) [19] since the spectrum redundancy caused by periodicity in the modulated signal results in correlation between widely separated frequency components [19], [20].

As a spectrum sensing scheme in CR, cyclostationary detection is especially appealing because it is capable of differentiating the primary signal from the interference and noise. Due to its noise rejection property, cyclostationary detection works even in very low SNR region, where the traditional signal detection method, such as the ED, fails. In [4], cyclostationary detectors have been demonstrated to enhance the detection capability, especially in the presence of noise power uncertainty. In [21], joint cyclostationary detection and optimal data fusion has been considered to improve the overall detection performance of CR networks. The FCC has suggested cyclostationary detectors as a useful alternative to enhance the detection sensitivity in CR networks [1].

Consider a typical digitally modulated signal of the form

$$
s(t)=\sum_{n} a(n) g\left(t-n T_{0}-t_{0}\right)
$$

where $T_{0}$ is the symbol period, $t_{0}$ is an unknown timing offset, and $g(t)$ is the shaping pulse. For simplicity, assume that the sequence $a(n)$ is stationary with zero mean and variance $\sigma_{a}^{2}$; then the time-varying autocorrelation function (TVAF) of $s(t)$ is defined as

$$
\begin{aligned}
R_{s}(t, \tau) & =E\left\{s(t+\tau) s^{*}(t)\right\} \\
& =\sum_{n} \sigma_{a}^{2} g\left(t+\tau-n T_{0}-t_{0}\right) g^{*}\left(t-n T_{0}-t_{0}\right) \\
& =\sum_{\alpha=k / T_{0}} R^{\alpha}(\tau) e^{j 2 \pi \alpha t}
\end{aligned}
$$

where

$R^{\alpha}(\tau)= \begin{cases}\frac{\sigma_{a}^{2} e^{j 2 \pi \alpha t_{0}}}{T_{0}} & \\ \times \int G^{*}(f+\alpha) G(f) e^{j 2 \pi f \tau} d f, & \alpha=\frac{k}{T_{0}} \\ 0, & \text { otherwise }\end{cases}$

and $G(f)$ is the Fourier transform of $g(t)$.

The function $R^{\alpha}(\tau)$ is called the cyclic autocorrelation function and $\alpha$ is called cyclic frequency. As indicated in (16), the CAF at a given cyclic frequency $\alpha$ determines the correlation between spectral components of the signal separated in frequency by an amount of $\alpha$. In general [19], the CAF of cyclostationary signals is nonzero only for integer multiples of a fundamental cyclic frequency $\alpha_{0}$. For the signal model given in (14), $\alpha_{0}=1 / T_{0}$. Thus, given $T_{0}$, the CAF can be utilized to determine the presence or absence of the primary signal by evaluating the values of $R^{\alpha}(\tau)$ at corresponding cyclic frequencies.

In practice, cyclostationary detection can be implemented in discrete time domain. Let $y(n), 0 \leq n \leq N-1$, denote the sampled received signal at the CR user; then the discrete-time CAF of the received signal at a cyclic frequency $\alpha$ can be estimated as [18]

$\widehat{R}^{\alpha}[l]=\frac{1}{N-l} \sum_{n=0}^{N-l-1} y(n+l) y^{*}(n) e^{-j 2 \pi \alpha n}, \quad 0 \leq l \leq L-1$

where $L$ is the number of lags. Then a vector is constructed as

$$
\begin{aligned}
& \widehat{\mathbf{r}}=[ \operatorname{Re}\left\{\widehat{R}^{\alpha}(1)\right\}, \operatorname{Re}\left\{\widehat{R}^{\alpha}(2)\right\}, \ldots, \operatorname{Re}\left\{\widehat{R}^{\alpha}(L)\right\} \\
&\left.\operatorname{Im}\left\{\widehat{R}^{\alpha}(1)\right\}, \operatorname{Im}\left\{\widehat{R}^{\alpha}(2)\right\}, \ldots, \operatorname{Im}\left\{\widehat{R}^{\alpha}(L)\right\}\right]
\end{aligned}
$$

where $\operatorname{Re}\{$.$\} and \operatorname{Im}\{$.$\} refer to the real and imaginary$ parts of a complex number, respectively. In [18], the statistical characteristics of $\widehat{\mathbf{r}}$ under $\mathcal{H}_{0}$ and $\mathcal{H}_{1}$ for a sufficiently large $N$ have been investigated; based on that, an algorithm has been developed to perform CAF-based cyclostationary detection. 
So far, we have assumed that the period of the primary signal $T_{0}$ is known to the CR user. This assumption is reasonable in the early stage of $\mathrm{CR}$ application since, in this stage, only limited spectrum bands, for example, the TV bands, are open to CR users and the characteristics of the primary signals are well known to the public. However, when CR is allowed to work in a wide spectrum band in the future, the periods of some modulated primary signals may be unknown to $\mathrm{CR}$ users. In this case, an exhaustive search of the cyclic frequencies is needed in cyclostationary detection. This means huge complexity and the loss of the ability to differentiate the primary signal from the interference that is also cyclostationary. Similar comments also apply to other spectrum sensing techniques that are based on the a priori knowledge of the primary signal.

\section{E. Pilot-Based Coherent Detection}

In practical communication systems, pilots are usually transmitted periodically to help receivers perform time or frequency synchronization, channel estimation, etc. These pilots, if known to CR users, can be utilized for coherent detection of the primary signal. Similar to cyclostationary detection, pilot-based coherent detection is capable of distinguishing the primary signal from the interference and noise. Therefore, it works even under a very low SNR region. Moreover, it has lower complexity and higher agility than statistics-based cyclostationary detection. Therefore, pilotbased coherent detection is always a preferred spectrum sensing scheme in practice.

It is well known that the optimal way for detection of a known signal is the matched filter (MF) detection [4] since it maximizes the received SNR. Here we give a brief review of the MF-based spectrum sensing scheme. Let the sampled received signal at the $\mathrm{CR}$ user be

$$
y(n)=\theta h p(n)+w(n), \quad 0 \leq n \leq N-1
$$

where $p(n)$ and $w(n)$ denote the pilot sequence and the white noise, respectively, $h$ denotes the quasi-static block fading channel from the primary transmitter to the $\mathrm{CR}$ user, and $\theta=0$ and $\theta=1$ denote the absence and presence of the primary signal, respectively. Define

$$
P_{p}=\frac{1}{N} \sum_{n=0}^{N-1}|p(n)|^{2}
$$

as the average power of the pilot signal. Then the instantaneous SNR within the current detection period is given by

$$
\gamma=\frac{|h|^{2} P_{p}}{\sigma_{n}^{2}}
$$

where $\sigma_{n}^{2}$ denotes the noise power.

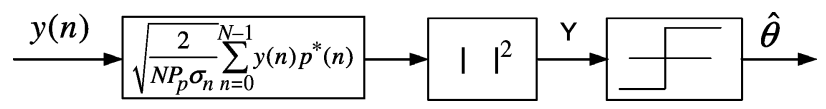

Fig. 4. Schematic representation of the MF detector.

Fig. 4 shows a schematic representation of the MF detector, which forms the test statistics as

$$
\begin{aligned}
Y & =\mid \sqrt{\left.\frac{2}{N P_{p} \sigma_{n}^{2}} \sum_{n=0}^{N-1} y(n) p^{*}(n)\right|^{2}} \\
& = \begin{cases}\left|\sqrt{\frac{2}{N P_{p} \sigma_{n}^{2}}} \sum_{n=0}^{N-1} w(n) p^{*}(n)\right|^{2}, & \mathcal{H}_{0} \\
\mid \sqrt{\frac{2 N P_{p}}{\sigma_{n}^{2}}} h & \\
+\left.\sqrt{\frac{2}{N P_{p} \sigma_{n}^{2}}} \sum_{n=0}^{N-1} w(n) p^{*}(n)\right|^{2}, & \mathcal{H}_{1}\end{cases}
\end{aligned}
$$

and makes the decision accordingly as

$$
\hat{\theta}= \begin{cases}\mathcal{H}_{1}, & \text { if } Y>\lambda \\ \mathcal{H}_{0}, & \text { if } Y<\lambda\end{cases}
$$

where the threshold $\lambda$ is chosen to satisfy a target falsealarm probability.

It can be easily shown that under the AWGN assumption, the test statistics of the MF detector $Y$ follows a central chi-square distribution with two degrees of freedom under $\mathcal{H}_{0}$ and a noncentral chi-square distribution with two degrees of freedom and a non-centrality parameter $\mu=2 \mathrm{~N} \gamma$ under $\mathcal{H}_{1}$, i.e.,

$$
f_{Y}(Y) \sim \begin{cases}\chi_{2}^{2}, & \mathcal{H}_{0} \\ \chi_{2}^{2}(\mu), & \mathcal{H}_{1}\end{cases}
$$

based on which the false-alarm probability and the detection probability for a given decision threshold can be obtained.

In the above discussion, we have assumed perfect timing with the transmitted pilot signal at the $\mathrm{CR}$ receiver. Since the CR user does not even know whether the primary signal exists, the perfect timing assumption may not be true in practice. Suppose there exists a maximum time offset of $L$ samples; then the MF detector has to perform an exhaustive search of the time offset by forming the following test statistics:

$$
Y_{\max }=\max _{0 \leq l \leq L}\left|\sqrt{\frac{2}{N P_{p} \sigma_{n}^{2}}} \sum_{n=0}^{N-1} y(n+l) p^{*}(n)\right|^{2} .
$$


As a result, the implementation complexity is increased and the detection performance is also degraded.

Recently, various pilot-based coherent detection schemes have been proposed for spectrum sensing in CR. In [22], FFT-based pilot energy and location detection schemes have been proposed for spectrum sensing in 802.22 WRAN. The two schemes are designed to utilize the pilot inserted in the ATSC digital TV signals for carrier frequency recovery at the receiver. In [23], both data and segment synchronization sequences in the ATSC digital TV signals have been utilized for spectrum sensing in 802.22 WRAN.

\section{F. Covariance-Based Detection}

The key idea behind covariance-based primary signal detection is that the primary signal received at the CR user is usually correlated because of the dispersive channels, the utility of multiple receive antennas, or even oversampling. Such correlation can be utilized by the CR user to differentiate the primary signal from white noise.

Specifically, covariance-based detector determines the presence or absence of the primary signal based on the covariance matrix of the received signal. Suppose that the received signal is given by

$$
y(n)=\theta s(n)+w(n), \quad 0 \leq n \leq N-1
$$

where $\theta=1$ and $\theta=0$ denote the presence and absence of the primary signal, respectively. Then the sample covariance matrix of the received signal can be estimated as

$$
\widehat{\mathbf{R}}_{y}=\frac{1}{N} \sum_{n=L-1}^{N-1} \mathbf{y}[n] \mathbf{y}^{\mathbf{H}}[n]
$$

where

$$
\mathbf{y}[n]=(y[n], y[n-1], \ldots, y[n-L+1])^{T} .
$$

It can be shown [24] that $\widehat{\mathbf{R}}_{y}$ converges in probability at

$$
\mathbf{R}_{y}=E\left\{\mathbf{y}[n] \mathbf{y}^{\mathbf{H}}[n]\right\}=\theta \mathbf{R}_{s}+\sigma_{n}^{2} \mathbf{I}_{L}
$$

where $\mathbf{R}_{s}$ is the $L \times L$ covariance matrix of the primary signal vector

$$
\mathbf{s}[n]=(s(n), s(n-1), \ldots, s(n-L+1))^{T}
$$

$\sigma_{n}^{2}$ is the noise power, and $\mathbf{I}_{L}$ is an $L \times L$ identity matrix.
Based on the sample covariance matrix of the received signal $\widehat{\mathbf{R}}_{y}$, various test statistics have been developed for detection of the primary signal, including the ratio of its maximum and minimum eigenvalues [24], the ratio of its diagonal and off-diagonal elements [25], and its maximum eigenvalue [26]. As an example, we introduce the first one in the following.

Let $\mu_{\max }$ and $\mu_{\min }$ denote the maximum and minimum eigenvalues of $\mathbf{R}_{y}$, respectively. Define $\mathbf{u}=\left(\mu_{\max }, \mu_{\min }\right)$; then, according to (29)

$$
\mathbf{u}= \begin{cases}\left(\sigma_{n}^{2}, \sigma_{n}^{2}\right), & \mathcal{H}_{0} \\ \left.\rho_{\max }+\sigma_{n}^{2}, \rho_{\min }+\sigma_{n}^{2}\right), & \mathcal{H}_{1}\end{cases}
$$

where $\rho_{\max }$ and $\rho_{\min }$ are the maximum and minimum eigenvalues of $\mathbf{R}_{s}$, respectively. Because of the correlation among the sampled primary signals $\rho_{\max }>\rho_{\min }$. Therefore, if there is no primary signal

$$
\frac{\mu_{\max }}{\mu_{\min }}=1
$$

otherwise

$$
\frac{\mu_{\max }}{\mu_{\min }}>1
$$

Based on the above heuristic, the eigenvalue-based detection algorithm can be formulated as follows [24].

1) Estimate the covariance matrix of the received signal according to (27).

2) Calculate the maximum and minimum eigenvalues of the sample covariance matrix as $\hat{\mu}_{\max }$ and $\hat{\mu}_{\text {min }}$, respectively, and compute the decision statistics

$$
Y=\frac{\hat{\mu}_{\max }}{\hat{\mu}_{\min }} .
$$

3) Obtain the final decision

$$
\hat{\theta}= \begin{cases}\mathcal{H}_{1}, & \text { if } Y>\lambda \\ \mathcal{H}_{0}, & \text { if } Y<\lambda\end{cases}
$$

In [24], the statistical characteristics of $\hat{\mu}_{\max }$ and $\hat{\mu}_{\text {min }}$ under $\mathcal{H}_{0}$ have been investigated. Based on that, a closed-from expression of the decision threshold $\lambda$ for a given false-alarm probability has been obtained. In general, it is tough to obtain a closed-form expression of the detection probability $P_{D}=\operatorname{Pr}\left\{\hat{\mu}_{\max }>\lambda \hat{\mu}_{\text {min }} \mid \mathcal{H}_{1}\right\}$. However, it can be calculated using numerical methods. 
It must be mentioned that the performance of covariance-based detection depends on the statistics of the received primary signal. In the extreme case that the primary signal appears like white noise, a covariance-based detector will fail. In practice, however, the correlation of the received primary signal can be created, for example, by utilizing multiple antennas at the $\mathrm{CR}$ receiver.

\section{G. Wavelet-Based Detection}

A wide spectrum band may consist of disjoint entities occupying different frequencies. Let $f_{0}, f_{1}, \ldots, f_{K}$ denote the frequency boundaries of $K$ disjoint spectrum bands occupying a total bandwidth of $B \mathrm{~Hz}$. The $\mathrm{CR}$ network needs to determine which of the spectrum bands is currently unoccupied so as to scavenge that particular band. In Section II-C, we have investigated energydetection-based wide-band spectrum sensing, in which the magnitude of the estimated PSD of the received signal is utilized to decide on the presence or absence of the primary signal in each spectrum band. By assuming that the power spectral characteristic is smooth within each subband but exhibits a discontinuous change between adjacent subbands, a novel approach has been proposed in [27] to identify and locate the spectrum holes by analyzing the irregularities in the estimated PSD with wavelet transform, an attractive mathematical tool for analyzing singularities and irregular structures of signals.

Let $y(n)$ denote a wide-band received signal at a CR user. It is a combination of signals from various primary users and is of the form

$$
y(n)=\sum_{k=1}^{K} \theta_{k} s_{k}(n)+w(n)
$$

where $\theta_{k}=1$ and $\theta_{k}=0$ denote the presence and absence of the primary signal in the $k$ th band, respectively, and $s_{k}(n)$ is the signal from the kth primary transmitter. In order to detect the presence of the $k$ th primary user, the PSD of $s_{k}(n), S_{k}(f)$ needs to be estimated. However, usually the frequency boundaries and even the value of $K$ are unknown to the CR network. In [27], a wavelet-based approach has been developed to estimate the number of subbands and the corresponding frequency boundaries. Once the region of support is determined, the PSD on each spectrum band can be estimated, based on which the presence of spectrum holes is detected.

Specifically, wavelet-based detection has been developed under the following assumptions [27].

1) The range of the entire spectrum band, i.e., the values of $f_{\min }\left(=f_{1}\right)$ and $f_{\max }\left(=f_{K}\right)$, is known to the CR network.

2) The number of licensed spectrum bands $K$ is unknown to the CR network.
3) The PSD of each occupied band is smooth and almost flat in its region of support, i.e., for all $k$, $S_{k}(f)=\alpha_{k}, f_{k} \leq f<f_{k+1}$.

4) The PSD of the noise process is flat throughout the whole bandwidth, i.e., $S_{n}(f)=N_{0}$ for all $f$.

With the above assumptions, the PSD of the received signal $y(n)$ can be expressed as

$$
S_{y}(f)=\sum_{k=1}^{K} \alpha_{k} \mathbb{1}\left(f_{k-1} \leq f<f_{k}\right)+N_{0}
$$

where $11(\cdot)$ refers to the indicator function. In practice, $S_{y}(f)$ needs to be estimated from $y(n)$. For example, in the periodogram approach [28], $S_{y}(f)$ is estimated as

$$
\widehat{S}_{y}(f)=\frac{1}{N} \sum_{k=0}^{N / L-1}\left|\sum_{l=0}^{L-1} y(l+k L) e^{-j \frac{2 \pi f_{l}}{L}}\right|^{2} .
$$

Given such an estimate of the PSD, it is of interest for the CR network to know which of the $\alpha_{k}$ s are nonzero.

In the wavelet-based approach developed in [27], an appropriate wavelet smoothing function $\phi(f)$ is first chosen to extract the features from the estimated PSD $\widehat{S}_{y}(f)$. Typical examples of $\phi(f)$ include the Gaussian function and the perfect reconstruction filter bank [29]. The continuous wavelet transform (CWT) of $\widehat{S}_{y}(f)$ is thus given by

$$
\widehat{W}_{y}(s ; f)=\widehat{S}_{y}(f) * \phi_{s}(f)
$$

where $*$ denotes convolution operation and $\phi_{s}(f)=$ $(1 / s) \phi(f / s)$ is the dilated version of $\phi(f)$ by a dyadic scale factor, $s=2^{j}, 1 \leq j \leq J$. Then the following proposition has been proved in [30].

Proposition: An estimate of the frequency boundaries $\hat{f}_{k} \mathrm{~s}$ can be obtained as the local maxima of the wavelet modulus $\widehat{W}_{y}^{\prime}(s ; f)$ for all values of $s,{ }^{6}$ where $\widehat{W}_{y}^{\prime}$ denotes the derivative of $\widehat{W}_{y}(s ; f)$ with respect to $f$, i.e.,

$$
\left\{\hat{f}_{k}, 1 \leq k \leq K\right\}=\arg \max _{f_{\min } \leq f \leq f_{\max }}\left|\prod_{j=1}^{J} \widehat{W}_{y}^{\prime}\left(2^{j} ; f\right)\right|
$$

where "max" denotes the local maximization operation.

Once the frequency boundaries are obtained based on (39), the number of subbands within the whole spectrum

${ }^{6}$ The frequency boundaries of the PSD have to be those $\hat{f}_{k}$ s that are local maxima of $\widehat{W}_{y}^{\prime}(s ; f)$ for all values of $s$ because random noises result in local maxima at some frequencies for isolated values of $s$. 
band $K$ can be determined accordingly. Afterwards, the PSD on each subband can be estimated as

$$
\widehat{\alpha}_{k}=\frac{1}{\hat{f}_{k}-\hat{f}_{k-1}} \int_{\hat{f}_{k-1}}^{\hat{f}_{k}} S_{y}(f) d f-N_{0}, \quad 1 \leq k \leq K .
$$

If $\alpha_{k} \approx 0$ for $k$, the $\mathrm{CR}$ user deduces that the corresponding spectrum band is unoccupied.

\section{H. Comments}

In the above discussion, we have introduced various basic spectrum sensing techniques. Different detectors are applicable to different scenarios and have different performance. The energy detector is the simplest and most robust detector. It does not require any a priori knowledge of the primary signal and works for any primary system. Therefore, it is suitable for detection of the primary signal about which the CR user knows nothing. In order to improve the detection performance, it is vital for a detector to be able to differentiate the primary signal from the interference and noise. In practice, such differentiation can be realized if some a priori knowledge of the primary signal is known to the CR user. Depending on what information the CR user knows about the primary signal, different detectors can be applied under different scenarios. A covariance-based detector is suitable when the primary signal is known as correlated; a cyclostationary detector is suitable when the period of the primary signal is known; a matched filter detector is suitable when the pilot signal of the primary system is known. Recently, probability-based spectrum sensing techniques have been proposed in [31] and [32] to utilize the statistical information on the primary user activity. The more the CR user knows about the primary signal, the better the detector works. As mentioned, in the early stage of CR application, the characteristics of the primary signal, such as the ATSC digital TV signal in 802.22 WRAN, are usually well known, and therefore pilot-based coherent detection or cyclostationary detection can be applied to improve the performance of spectrum sensing.

\section{SPECTRUM SENSING: COOPERATIVE DETECTION}

As indicated before, spectrum sensing plays a critical role in CR networks. However, multipath fading and shadowing, the fundamental characteristics of wireless channels, present a major challenge of spectrum sensing. If the primary signal is deeply faded or blocked by large obstacles in the environment, then the power of the received primary signal will be too weak to detect its presence. Fortunately, the impact of multipath fading and shadowing can be mitigated by the spatial diversity in multiuser CR networks. In [33], it has been shown that the detection capability of the CR network can be improved by letting multiple $\mathrm{CR}$ users take turns to sense the channel instead of designating a fixed CR user for spectrum sensing. Since multipath fading varies significantly on the scale of halfwavelength and shadowing varies significantly on the scale of 20-500 m depending on the environment, the probability that multiple CR users are experiencing deep fading or are blocked by obstacles simultaneously is rather low. Therefore, cooperation among CR users will effectively improve the performance of spectrum sensing. It has been demonstrated that cooperation can improve the detection performance [34], relax the sensitivity requirements [35], and decrease the detection time required [36], [37]. In this section, we will present various cooperative spectrum sensing techniques that have been proposed recently for centralized and decentralized CR networks, respectively.

\section{A. Centralized CR Networks}

Cooperative spectrum sensing in a centralized $\mathrm{CR}$ network consists of a base station or access point and a number of CR users. In this network, each CR user sends its sensing information to the base station via the common control channels while the base station combines the sensing information and makes a decision on the presence or absence of the primary signal. For simplicity, we assume that sensing information is sent from the CR users to the base station free of error. Generally, the sensing information combination at the base station can be categorized by soft combination and hard combination techniques.

Soft Combination: In soft combination, the CR users send their original sensing data to the base station without quantization. While soft combination requires large overhead to feedback the sensing data, it has excellent detection performance.

In [3], Haykin has recommended a multitaper-method singular-value-decomposition (MTM-SVD) based cooperative scheme to estimate the interference temperature of the radio environment. As demonstrated below, this scheme is essentially a soft-combination-based cooperative spectrum sensing scheme. Consider an $M$-user cooperative CR network, where the multitaper method is applied at each CR user for wide-band spectral analysis. In the MTMSVD method, the $m$ th $(1 \leq m \leq M)$ CR user computes its $k$ th eigenspectrum over the frequency of interest by

$$
Y_{k}^{(m)}(f)=\sum_{n=1}^{N} w_{k}(n) y_{m}(n) e^{-j 2 \pi f n}, \quad 1 \leq k \leq K
$$

where $y_{m}(n)$ is the received signal at the $m$ th CR user and $w_{k}(n)$ is the $k$ th Slepian sequence used for multitaper 
spectral analysis. Then each CR user sends its eigenspectrum vector

$$
\begin{aligned}
& \mathbf{Y}_{m}(f)=\left(Y_{1}^{(m)}(f), Y_{2}^{(m)}(f), \cdots, Y_{K}^{(m)}(f)\right), \\
& 1 \leq m \leq M
\end{aligned}
$$

to the base station. Then an $M \times K$ eigenspectrum matrix is constructed at the base station as

$$
\mathbf{A}(f)=\left[\begin{array}{cccc}
\omega_{1} Y_{1}^{(1)}(f) & \omega_{1} Y_{2}^{(1)}(f) & \cdots & \omega_{1} Y_{K}^{(1)}(f) \\
\omega_{2} Y_{1}^{(2)}(f) & \omega_{2} Y_{2}^{(2)}(f) & \cdots & \omega_{2} Y_{K}^{(2)}(f) \\
\vdots & \vdots & \ddots & \vdots \\
\omega_{M} Y_{1}^{(M)}(f) & \omega_{M} Y_{2}^{(M)}(f) & \cdots & \omega_{2} Y_{K}^{(M)}(f)
\end{array}\right]
$$

where $\omega_{m}$ denotes the weight allocated to the $m$ th CR user to take its geographic environment into account.

By combining the eigenspectrum vectors of different CR users, the MTM-SVD method is capable of utilizing the spatial diversity in wireless channels to improve the performance of spectrum sensing. But this is not the full story yet. The eigenspectrum vector from each $\mathrm{CR}$ user consists of the primary signal and noise. For different CR users, the noise part is independent while the primary signal part is correlated. In light of this, the MTM-SVD method tries to utilize the correlation in the primary signal part of the eigenspectrum vectors from different CR users to improve the detection performance. This is accomplished by applying the singular value decomposition to the eigenspectrum matrix

$$
\mathbf{A}(f)=\sum_{k=1}^{K} \sigma_{k}(f) \mathbf{u}_{k}(f) \mathbf{v}_{k}^{H}(f)
$$

where $\sigma_{k}(f)$ is the kth singular value of $\mathbf{A}(f), \mathbf{u}_{k}(f)$ is the associated left singular vector, and $\mathbf{v}_{k}(f)$ is the associated right singular vector. Then the base station makes a decision on the presence or absence of the primary signal according to the largest singular value of $\mathbf{A}(f)$. Due to the accuracy of the mutltitaper spectral analysis and the denoising effect of the SVD, the MTM-SVD-based cooperative spectrum sensing scheme can achieve a nearly optimal performance [3].

In the MTM-SVD method, the CR user needs to send a $\mathrm{K}$-dimensional eigenspectrum vector to the base station while the base station needs to perform SVD operation on the constructed eigenspectrum matrix. Therefore, it requires lots of feedback overheads and has high computational complexity. In [38], various soft-combinationbased cooperative energy detection schemes with low complexity have been investigated. In these schemes, each CR user sends its observed energy of the received signal to the base station while the base station decides on the presence or absence of the primary signal based on a weighted summation of these energies

$$
Y_{c}=\sum_{m=1}^{M} \omega_{m} Y_{m}
$$

where $Y_{m}$ is the observed energy of the $m$ th $\mathrm{CR}$ user given by (6) and $\omega_{m}$ is the associated weight.

In [38], an optimal combination (OC) scheme of the observed energies that maximizes the detection probability for a given false-alarm probability has been proposed based on the Gaussian primary signal assumption. In the OC scheme, the weight corresponding to each CR user is based on the instantaneous SNR of that user. Although the OC scheme works well as a performance benchmark for various soft combination schemes, it needs the instantaneous channel information of the CR users and may be impractical in certain environments. In contrast, the equal-gain combination (EGC) of the observed energies does not need any channel information and provides a reasonable performance as well [38]. Therefore, EGC is a feasible soft combination scheme in practice.

Hard Combination: Although soft combination schemes have a good detection performance, they require a lot of overhead to feedback the observation, which sacrifices the spectral efficiency. Recently, hard combination schemes have been proposed to achieve a tradeoff between performance and complexity [34], [38]. In hard combination schemes, the CR users send quantized sensing information to the base station. While local hard decision at the CR users causes information loss and performance degradation, it greatly reduces the amount of feedback. For simplicity, here we only consider energy detection; extension to other basic spectrum sensing techniques introduced in Section II is straightforward.

The simplest hard combination scheme is the one-bit counting scheme, in which each $\mathrm{CR}$ user sends one-bit information to the base station regarding whether its observed energy is above a predetermined threshold. If there are $K \mathrm{CR}$ users out of $M$ above this threshold, the primary signal will be declared present. So this decision criterion is also called $K$-out-of- $M$ rule [39]. While the optimal value of $K$ in the one-bit counting scheme generally depends on the type of channel CR users experience, it has been demonstrated in [40] that the 1-out-of-M (OR) rule has the best detection performance under most practical fading channels.

In the conventional one-bit counting scheme, there is only one threshold dividing the observed energy into two regions with equivalent weights $w_{0}=0$ and $w_{1}=1$, 


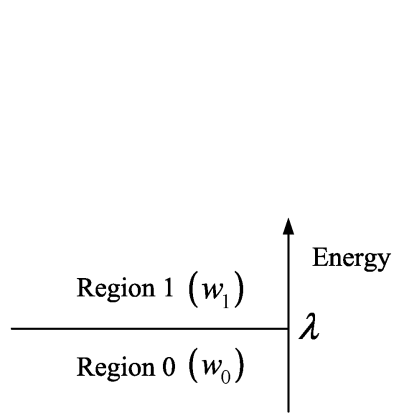

(a)

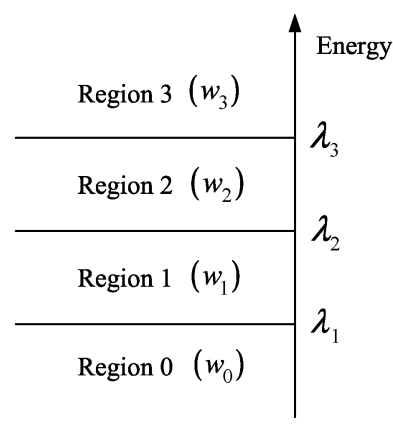

(b)
Fig. 5. Principles of hard combination schemes. (a) One-bit counting scheme and (b) two-bit hard combination scheme.

respectively, as shown in Fig. 5(a). As a result, all of the $\mathrm{CR}$ users above this threshold are allocated the same weight regardless of the possible significant differences in their observed energies. Intuitively, a better detection performance can be achieved if we divide the observed energy into more regions, allocating larger weights to the upper regions and smaller weights to the lower regions. Based on the above heuristic, a new two-bit hard combination scheme has been proposed in [38], the principle of which is shown in Fig. 5(b). In this scheme, three thresholds $\lambda_{1}, \lambda_{2}$, and $\lambda_{3}$ divide the observed energy into four regions with different weights $w_{0}, w_{1}, w_{2}$ and $w_{3}$. Therefore, each CR user needs to feedback twobit information to indicate the region of its observed energy. Different from the one-bit counting scheme, the two-bit hard combination scheme calculates a weighted summation of the numbers of CR users falling in different regions. In [38], the optimal partition of the regions and weight allocation for the two-bit hard combination scheme have been investigated. It has been demonstrated in [38] that the two-bit hard combination scheme exhibits comparable performance with the EGC soft combination scheme despite its having much less complexity and overhead.

\section{B. Decentralized CR Networks}

Cooperative communication schemes with orthogonal transmission have been proposed in [41] and [42]. It has been shown in [41] that the amplify-and-forward (AF) cooperation protocol, in which one user acts as a relay for the other and amplifies and forwards the signal received from its partner without any further processing, achieves full cooperative diversity. Recently, cooperative spectrum sensing in a decentralized CR network equipped with this AF cooperation protocol has been investigated in [36] and [37].

Fig. 6 shows a schematic representation of the AF cooperation scheme in a decentralized $\mathrm{CR}$ network. In the figure, $P$ denotes the primary user and $C R$ user $U_{1}$ is sending data to $\mathrm{CR}$ user $U_{3}$, while $\mathrm{CR}$ user $U_{2}$ acts as an $\mathrm{AF}$ relay for $U_{1}$. The $\mathrm{AF}$ cooperation scheme consists of two stages or time slots [36]: in the first time slot, $t_{1}$, $U_{1}$ transmits while $U_{2}$ listens; in the second time slot, $t_{2}$, $U_{2}$ transmits while $U_{1}$ keeps silent. Thus orthogonal transmission of $U_{1}$ and $U_{2}$ is guaranteed. Since continuous spectrum sensing is required during the process of secondary communication between the CR users, actually $U_{1}$ does not idle the second time slot away. Instead, $U_{1}$ listens to its partner $U_{2}$ and decides whether the received signal contains the primary signal in the second time slot.

According to the AF cooperation scheme, the received signal at $U_{2}$ in the first time slot is given by

$$
y_{2}\left(t_{1}\right)=\theta h_{p 2} p\left(t_{1}\right)+h_{12} s\left(t_{1}\right)+w_{2}\left(t_{1}\right)
$$

where $t_{1}$ denotes the first time slot, $p\left(t_{1}\right)$ and $s\left(t_{1}\right)$ denote the primary signal and the transmitted signal of $U_{1}$, respectively, $h_{p i}$ and $h_{i j}, 1 \leq i, j \leq 2$, denote the channel gain between $P$ and $U_{i}$, and between $U_{i}$ and $U_{j}$, respectively, $w_{2}\left(t_{1}\right)$ denotes the noise at $U_{2}$, and $\theta=1$ and $\theta=0$ denote the presence and absence of the primary signal, respectively.

The received signal at $U_{1}$ in the second time slot is given by

$$
y_{1}\left(t_{2}\right)=g h_{21} y_{2}\left(t_{1}\right)+\theta h_{p 1} p\left(t_{2}\right)+w_{1}\left(t_{2}\right)
$$

where $g$ denotes the amplifying gain of the relay and $h_{21}=h_{12}$ since the channels are assumed to be reciprocal. After cancelling $s\left(t_{1}\right)$ from $y_{1}\left(t_{2}\right)$, the left signal at $U_{1}$ under the cooperation case is given by

$$
\hat{y}_{1}\left(t_{2}\right)=\theta\left[h_{p 1} p\left(t_{2}\right)+g h_{21} h_{p 2} p\left(t_{1}\right)\right]+w_{1}\left(t_{2}\right)+g h_{21} w_{2}\left(t_{1}\right) .
$$

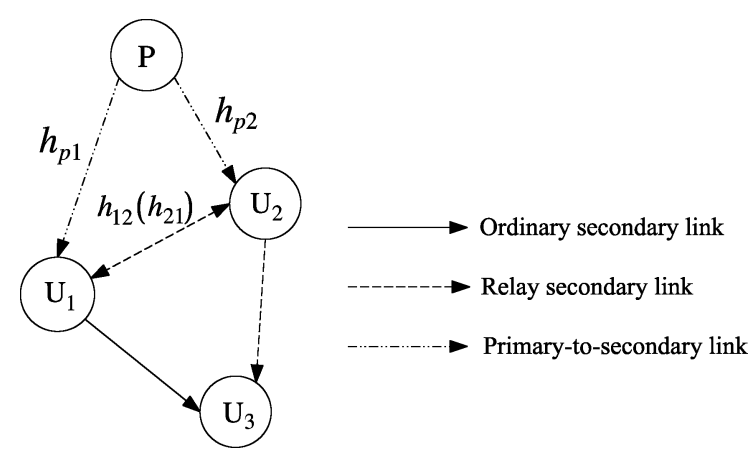

Fig. 6. Schematic representation of the AF cooperation scheme in a decentralized CR network. 
In contrast, the received signal at $U_{1}$ under noncooperation case is given by

$$
y_{1}(t)=\theta h_{p 1} p(t)+w(t) .
$$

Denote $P_{D, C}$ and $P_{D, N}$ to be the detection probabilities of $U_{1}$ under cooperation and noncooperation cases for a given false-alarm probability, respectively. In [36], it has been demonstrated that whether $P_{D, C}$ is greater than $P_{D, N}$ depends on the channel gain between the primary user and the relay $h_{p 2}$, the amplifying gain of the relay $g$, and the channel gain between the source CR user and the relay $h_{21}$. In other words, AF cooperation improves the performance of spectrum sensing only under certain scenarios. Therefore, appropriate pairing of cooperative CR users is needed to optimize the overall detection capability of the CR network. In [37], an efficient pairing algorithm has been developed for a decentralized CR network, and the corresponding performance analysis has also been delivered.

\section{SPECTRUM SCULPTING}

Detecting the presence of primary users in a given frequency band is the first step in the operation of a CR network. In order to utilize the available bands, which change with time, CR operators need to avoid introducing interferences to the primary users as well as ensure proper reception of secondary signal. The measures a working CR network may take to accomplish these goals include: band-dropping; tone-nulling, particularly in an OFDM framework; signal-shaping, commonly referred to as spectrum shaping; and active interference cancelation.

When adjacent channel interferences to and from the primary users occur, the CR network may simply drop the band. This will require the $\mathrm{CR}$ system to adopt and incorporate frequency diversity in order to maintain an uninterrupted communication, something OFDM is ready to support. Band-dropping, although resulting in inefficient use of the spectrum, has the advantage of much simplified and eased handling of signal digitization and quantization right after the intermediate-frequency stage of the demodulation process [43]. Tone-nulling is similar to band-dropping and uses more advanced techniques to suppress the side lobes, at a higher resolution in terms of the frequency selectivity. Active interference cancellation as proposed in [44] requires the computation of canceling tones for each affected OFDM symbol to create additional notch depth. In this paper, we focus on signal or spectrum shaping.

As mentioned, in any CR network, the bandwidth available for transmission typically varies with time because the CR users need to avoid those (spectrum) regions that are currently occupied by the primary users. As a result, the $\mathrm{CR}$ users must be equipped with the capability to shape the spectrum of the transmitted signal so as to avoid interference with the primary users. More precisely, let $B_{1}, B_{2}, \ldots, B_{N}$ denote the available spectrum bands for the CR network and let $s(t)$ denote the transmitted signal of any CR user with PSD $S(f)$; then it is required that

$$
\operatorname{support}(S) \subseteq \bigcup_{k=1}^{N} B_{k}
$$

where support $(S)$ denotes the set of all frequencies $f$ satisfying that $S(f)>\eta$ with $\eta$ being a predefined threshold. To meet this requirement, multicarrier communication techniques and TDCS have been proposed recently for CR transmission.

\section{A. Multicarrier-Based Cognitive Radio}

Multicarrier communication techniques have been proposed as candidates for the physical layer of CR systems because they can provide a flexible spectrum shape that fills the gaps in the available bandwidth without causing interference to the primary users. This is commonly referred to as spectrum pooling [45]. Essentially, the idea behind spectrum pooling is to merge available spectrum holes into a virtual band for CR users. This is especially important in high-data-rate applications that typically require a large bandwidth. Another advantage of multicarrier techniques as physical layer candidates of CR systems is that the multicarrier processing structure employed for signal transmission and reception can be also utilized for wide-band spectral analysis. Consequently, spectrum sensing can be performed without any additional cost.

OFDM, the most popular multicarrier technique, has been proposed as the first candidate for the physical layer of CR systems [45]. Since the OFDM technique has an inherent capability to combat multipath fading and avoid the intersymbol interference, it is especially suitable for wide-band wireless communications. As a mature technique that has been intensively studied for the past decades, OFDM multicarrier technique is a natural choice for CR transmission.

In OFDM-based spectrum pooling CR networks, by nulling the appropriate subcarriers, the transmitter can avoid the spectrum regions currently used by the licensed users or other cognitive users. Let $F=\left\{f_{k}, 1 \leq k \leq N\right\}$ denote the total set of frequencies that may be used for CR transmission and $D_{t} \subseteq F$ denote the available set of frequencies at the current time slot $t$; then the corresponding time-domain OFDM signal of the CR transmitter is given by

$$
s(t)=g(t) \sum_{f_{k} \in D_{t}} a_{k} e^{j 2 \pi f_{k} t}, \quad 0 \leq t \leq T_{S}
$$




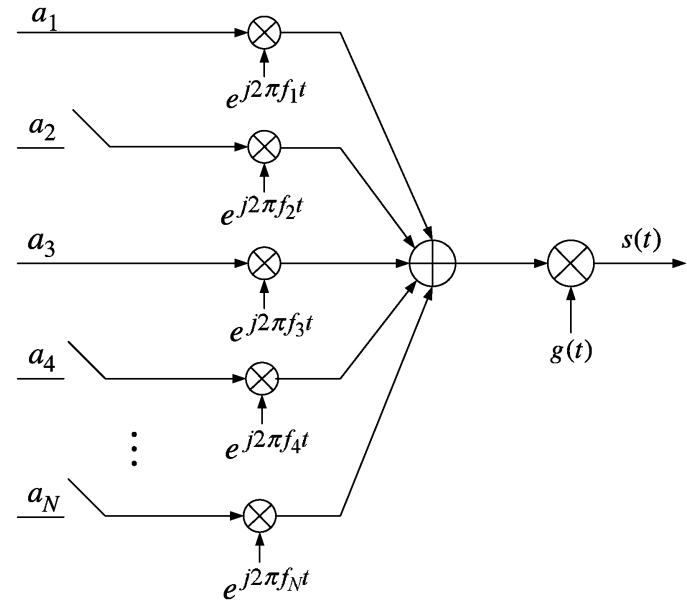

Fig. 7. Schematic representation of the OFDM-based CR transmitter.

where $g(t)$ is the time-domain windowing function, $a_{k}$ denotes the symbol to be transmitted at the $k$ th subchannel, and $T_{S}$ denotes the OFDM symbol duration. Fig. 7 illustrates a schematic representation of the OFDMbased CR transmitter, in which $f_{1}, f_{3} \in D_{t}$ for the current time slot $t$.

OFDM-based spectrum pooling networks have been discussed in [45] and [46]. In [45], various potential problems in employing OFDM-based spectrum pooling networks have been identified, among which we focus on the mutual interference between the $\mathrm{CR}$ users and the primary users because it is critical in secondary CR networks that are supposed to work on a noninterference basis.

The conventional OFDM system divides the whole bandwidth into a number of orthogonal subchannels, and therefore there is no cross-interference between different subchannels. For OFDM-based CR networks, however, it is important to note that though the CR network employs OFDM technology, it is not necessary that the primary network also does so. Even if the primary network also employs OFDM modulation with the same subcarrier spacing as the CR network, it is difficult to achieve synchronization between the two different systems. Therefore, there inevitably exists mutual interference between the primary and the secondary systems.

Interference to Primary System: In a typical OFDM system prototype, $g(t)$ in (51) is a rectangular windowing function, i.e.,

$$
g(t)= \begin{cases}1, & 0 \leq t \leq T_{S} \\ 0, & \text { otherwise }\end{cases}
$$

Suppose the signal sequence $\left\{a_{k}\right\}$ on the $k$ th subchannel is stationary with zero mean and variance $\sigma_{k}^{2}$; then the PSD of the random signal on the kth frequency $s_{k}(t)=$ $a_{k} g(t) e^{j 2 \pi f_{k} t}$ is given by [47]

$$
S_{k}(f)=\sigma_{k}^{2} T_{S}\left(\frac{\sin \left(\pi\left(f-f_{k}\right) T_{S}\right)}{\pi\left(f-f_{k}\right) T_{S}}\right)^{2}
$$

which consists of a squared sinc function and, hence, has large side lobes. It has been shown in [48] that about $13.16 \%$ of the total power of $S_{k}(f)$ leaks to the first eight adjacent subchannels, which may cause severe interference to adjacent primary users.

Different signal-processing techniques have been developed recently to reduce the power leakage of the OFDM-based secondary system to the primary system. In [48], it has been proposed to replace the original rectangular window in (52) with a raised-cosine window so as to reduce the power leakage in the side lobes. Another more effective scheme [48] is for CR users to deactivate the subchannels that are adjacent to the primary bands. The insertion of special cancellation carriers has been proposed in [49] to counteract the side lobes of the secondary signals. While all of the above schemes reduce the interference to the primary system at the expense of a reduced spectral efficiency, an efficient scheme has been proposed in [50] that reduces the side lobes by appropriately weighting the multiple phase-shift keying modulated OFDM subcarriers and, hence, does not consume any additional bandwidth.

Interference to Secondary System: Because of the nonorthogonality between the primary and the secondary subchannels, the primary system also causes interference to the OFDM-based secondary system. An OFDM-based CR receiver truncates the received signal according to the OFDM symbol duration $T_{S}$ and then performs an FFT operation on it. Suppose the discrete-time primary signal on the $k$ th subchannel is $p_{k}[n]$ with PSD $S_{P}\left(e^{j \omega}\right)$; then the truncated primary signal is given by

$$
\hat{p}_{k}[n]=p_{k}[n] g[n]
$$

where $g[n]$ is a rectangular windowing function with the same length as the OFDM block $N$.

Denote the PSD of $\hat{p}_{k}[n]$ as $\hat{S}_{P}\left(e^{j \omega}\right)$; then $\hat{S}_{P}\left(e^{j \omega}\right)$ can be regarded as an estimate of $S_{P}\left(e^{j \omega}\right)$ through periodogram approach with the period $N$, and therefore [51]

$$
\begin{aligned}
E\left\{\hat{S}_{P}\left(e^{j \omega}\right)\right\} & =S_{P}\left(e^{j \omega}\right) * W\left(e^{j \omega}\right) \\
& =\frac{1}{2 \pi} \int_{-\pi}^{\pi} S_{P}\left(e^{j \omega}\right) \frac{\sin ^{2}(N(\omega-\phi) / 2)}{\sin ^{2}((\omega-\phi) / 2)} d \phi
\end{aligned}
$$


where

$$
W\left(e^{j \omega}\right)=\frac{1}{N}\left|G\left(e^{j \omega}\right)\right|^{2}
$$

and $G\left(e^{j \omega}\right)$ is the discrete Fourier transform of $g[n]$. Therefore, $W\left(e^{j \omega}\right)$ can be equivalently regarded as the power spectral function of the time-domain window $g[n]$.

Equation (55) indicates that the PSD of the truncated primary signal is the convolution of the PSD of the original primary signal and the power spectral function of $g[n]$. Therefore, even if $S_{P}\left(e^{j \omega}\right)$ is ideally rectangular-shaped, there will still be power leakage to the secondary bands because of the signal truncation by the windowing function. One example in [48] has shown that there is about $2.32 \%$ power leakage to the first eight adjacent subchannels. According to (55), one way to reduce the power leakage is to apply a better time-domain windowing function, such as the raised-cosine function, to truncate the received signal at the OFDM-based CR receiver.

Other Issues in OFDM-Based Cognitive Radio: When OFDM is applied for CR transmission, only a part of subchannels that are not currently occupied by the primary system can be utilized, which is different from the conventional OFDM modulation. In light of this, efficient pruning algorithms have been proposed in [52] to reduce the processing time involved in FFT computation in practical OFDM-based CR networks. In [53], a discontiguous OFDM protocol has been proposed for dynamic spectrum access in idle TV channels. In [54], a novel low-complexity algorithm has been proposed for reducing the peak-to-average power ratio in OFDM-based cognitive radios. In general, the research in this area has been primarily focusing on OFDM technology suited to CR networks and reduction of adjacent channel interference.

Filter Bank Multicarrier Techniques for CR Transmission: OFDM modulation is an attractive scheme for CR transmission from the implementation point of view. However, as discussed above, OFDM-based cognitive radio suffers from severe mutual interference between the primary and the secondary systems. Although some interference mitigation algorithms have been proposed in the literature, they either sacrifice the spectral efficiency or are only applicable to certain scenarios. While it has been proposed that the FFT operation employed in an OFDM receiver can be utilized for power spectrum estimation in energydetection-based wide-band spectrum sensing, it is essentially periodogram spectral estimation, which is known as a biased and inconsistent estimator [3]. Therefore, such a power spectrum estimate suffers from a low SDR and fails to detect the presence of low-power primary signals.

Recently, filter bank multicarrier communication techniques, including the OFDM-OQAM, CMT, and
FMT, have been proposed as alternative physical-layer candidates of CR systems [16]. From the theory point of view, all of the three filter-bank multicarrier techniques share the same prototype filter: the root-Nyquist filter, an ideal low-pass filter without side lobes. From the implementation point of view, the side lobes of their prototype filters can be made arbitrarily small with relatively low complexity. Therefore, when applied to CR transmission, such filter-bank multicarrier techniques can effectively mitigate the mutual interference between the primary and the secondary systems without sacrificing the spectral efficiency. Moreover, when applied to power spectrum estimation, these filter banks can achieve a similar SDR as the multitaper method with a lower complexity, as discussed in Section II. Because of the above advantages, filter-bank multicarrier techniques have been recommended in [16] as candidates to replace the conventional OFDM multicarrier technique for CR transmission.

\section{B. TDCS-Based Cognitive Radio}

The TDCS was initially proposed in [55] for military communications. In a TDCS implementation, the transmitted wide-band waveform is synthesized flexibly according to the unoccupied and uncontaminated spectrum bands so as to avoid interference to and from other communication systems. Essentially, TDCS is a spread spectrum communication system with low transmit power. Therefore, TDCS is suitable for CR networks that are supposed to work on a noninterference basis. Actually, initial results presented in [56] have suggested that TDCS is a potential candidate for $\mathrm{CR}$ transmission. In this section, we give a brief introduction to the basic TDCS implementation and some initial research results regarding TDCS-based cognitive radio.

Basic TDCS Implementation: Fig. 8 shows the transmitter structure of a basic TDCS. As shown in the figure, the TDCS transmitter needs to observe the radio surrounding before each data transmission so as to identify those spectrum bands that have been occupied by the primary system or contaminated by adverse interferers. Various power spectrum estimation techniques introduced in Section II-C can be applied here for spectrum analysis. From the spectrum estimation $P(\omega)$, the spectrum magnitude $A(\omega)$ is obtained by

$$
A(\omega)= \begin{cases}1, & \text { if } P(\omega)<\lambda \\ 0, & \text { otherwise }\end{cases}
$$

where $\lambda$ is the threshold to determine whether a spectrum band is usable or not. Then the spectrum of the basis function for the TDCS is obtained by

$$
F_{b}(\omega)=C A(\omega) e^{j \phi(\omega)}
$$




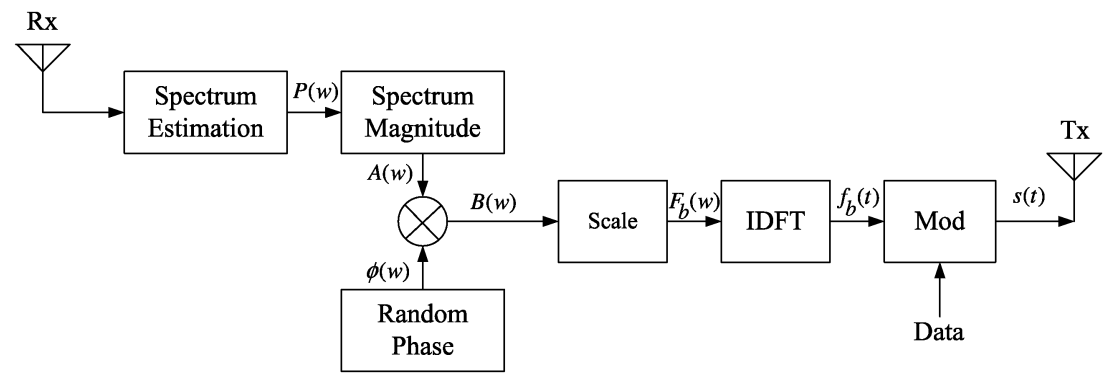

Fig. 8. Transmitter structure of a basic TDCS.

where $C$ is the normalization factor to ensure $F_{b}(\omega)$ has unit energy and $\phi(\omega)$ is the random phase to ensure that the generated waveform $F_{b}(\omega)$ has similar properties as white noise to avoid wiretapping. Afterwards, the timedomain basis function $f_{b}(t)$ is obtained as the inverse Fourier transform of $F_{b}(\omega)$. Once the basis function is obtained, different modulation schemes, such as the antipodal modulation and binary cyclic shift keying [57], can be applied for data transmission.

Initial Research Results: In [57], various potential CR transmission schemes, including TDCS, OFDM, and multicarrier code-division multiple access (MC-CDMA), have been discussed. In particular, the fundamental differences of TDCS relative to OFDM and MC-CDMA have been investigated. In [58], it has been observed that the OFDMbased TDCS is especially suitable for random spectrum availability in CR. Enlightened by this phenomena, an interleaved OFDM-based TDCS has been proposed in [58] that has excellent performance especially in the scenario when the percentage of available spectrum bands is low.

\section{CONCLUSION}

In this paper, we have discussed the practical issues involved to build a cognitive radio network from perspective of signal processing. We have identified two fundamental capabilities for any CR network to adapt to its environment and provide resilience under adverse conditions.

1) Spectrum sensing: The $C R$ network needs to continuously monitor the occupied spectrum for possible presence of the licensed users. We have discussed various basic spectrum sensing techniques that exploit null, minimal, or full knowledge of the primary signal characteristics. We have also addressed various cooperative spectrum sensing techniques that can effectively improve the overall detection capability of a CR network by taking advantage of the spatial diversity in wireless channels.

2) Spectrum sculpting: In order to avoid interference with the primary users, the physical layer of the CR user needs to continuously adapt the spectrum of the transmitted signal. This is challenging in the $\mathrm{CR}$ environment where the available spectrum varies dynamically with time. In this paper, we have considered multicarrier techniques and TDCS as possible candidates for the physical layer of a CR network. Both of them are able to flexibly switch the transmission off in the subset of carriers occupied by the primary users.

Due to space limitations, we are unable to deal with dynamic spectrum access in this paper. To ensure the longevity of the occupancy, the $\mathrm{CR}$ network needs to choose that portion of spectrum from the available bandwidth that has the least probability of occupancy by the primary users in the near future. From a signal processing perspective, smart-scan algorithms need to be developed to adapt to varying primary user occupancy and improve the overall spectrum efficiency of the CR network. Readers can refer to [59]-[62] for related materials in this area.

\section{REFERENCES}

[1] FCC, "Spectrum Policy Task Force report," ET Docket 02-155, Nov. 2002.

[2] I. J. Mitola, "Software radios: Survey, critical evaluation and future directions," IEEE Aerosp. Electron. Syst. Mag., vol. 8, pp. 25-31, Apr. 1993.

[3] S. Haykin, "Cognitive radio: Brain-empowered wireless communications," IEEE J. Sel. Areas Commun., vol. 23, pp. 201-220, Feb. 2005.
[4] D. Cabric, S. M. Mishra, and R. W. Brodersen, "Implementation issues in spectrum sensing for cognitive radios," in Proc. Asilomar Conf. Signals, Syst., Comput., Nov. 2004, vol. 1, pp. 772-776.

[5] G. D. Zhao, J. Ma, Y. G. Li, T. Wu, Y. H. Kwon, A. Soong, and C. Y. Yang, "Spatial spectrum holes for cognitive radio with directional transmission," in Proc. IEEE Globecom'08, New Orleans, Louisiana, Nov. 2008.
[6] A. Sahai, N. Hoven, and R. Tandra, "Some fundamental limits in cognitive radio," in Proc. Allerton Conf. Commun., Contr. Comput., Urbana, IL, Oct. 2004.

[7] A. Ghasemi and E. S. Sousa, "Spectrum sensing in cognitive radio networks: Requirements, challenges and design trade-offs," IEEE Commun. Mag., vol. 46, pp. 32-39, Apr. 2008.

[8] H. V. Poor, An Introduction to Signal Detection and Estimation, 2nd ed. Berlin, Germany: Springer-Verlag, 1994. 
[9] F. F. Digham, M. S. Alouini, and M. K. Simon, "On the energy detection of unknown signals over fading channels," in Proc. IEEE ICC, Anchorage, AK, May 2003, vol. 5, pp. 3575-3579.

[10] I. S. Gradshteyn and I. M. Ryzhik, Table of Integrals, Series, and Products, 6th ed. San Diego, CA: Academic, 2000.

[11] B. Farhang-Boroujeny, "Filter bank spectrum sensing for cognitive radios," IEEE Trans. Signal Process., vol. 56, pp. 1801-1811, May 2008.

[12] P. D. Welch, "The use of FFT for the estimation of power spectra: A method based on time averaging over short modified periodograms," IEEE Trans. Audio Electroacoust., no. 2, pp. 70-73, 1967.

[13] J. Lim and A. V. Oppenheim, Advanced Topics in Signal Processing. Englewood Cliffs, NJ: Prentice-Hall, 1988.

[14] D. J. Thomson, "Spectrum estimation and harmonic analysis," Proc. IEEE, vol. 20, pp. 1055-1096, Sep. 1982

[15] P. Stoica and T. Sundin, "On nonparametric spectral estimation," Circuits, Syst., Signal Process., vol. 16, pp. 169-181, 1999.

[16] B. Farhang-Boroujeny and R. Kempter, "Multicarrier communication techniques for spectrum sensing and communication in cognitive radios," IEEE Commun. Mag., vol. 46, pp. 80-85, Apr. 2008.

[17] R. Tandra and A. Sahai, "Fundamental limits on detection in low SNR under noise uncertainty," in Proc. Wireless Commun. Symp. Signal Process., Jun. 2005.

[18] A. V. Dandawate and G. B. Giannakis, "Statistical tests for presence of cyclostationarity," IEEE Trans. Signal Process. vol. 42, pp. 2355-2369, Sep. 1994

[19] W. A. Gardner, "Exploitation of spectral redundancy in cyclostationary signals," IEEE Signal Process. Mag., vol. 8, pp. 14-36, Apr. 1991.

[20] Y. G. Li and Z. Ding, "ARMA system identification based on second-order cyclostationarity," IEEE Trans. Signal Process. vol. 42, pp. 3483-3494, Dec. 1994.

21] C. R. C. M. da Silva, B. Choi, and K. Kim, "Distributed spectrum sensing for cognitive radio systems," in Proc. ITA Workshop 2007.

[22] C. Cordeiro, M. Ghosh, D. Cavalcanti, and K. Challapali, "Spectrum sensing for dynamic spectrum access of TV bands," in Proc. IEEE CROWNCOM, Orlando, FL, Aug. 2007, pp. 225-233.

[23] H. S. Chen, W. Gao, and D. G. Daut, "Signature based spectrum sensing algorithm for IEEE 802.22 WRAN," in Proc. IEEE ICC, Glasgow, Scotland, Jun. 2007, pp. 6487-6492.

24] Y. Zeng and Y. C. Liang, "Maximum-minimum eigenvalue detection for cognitive radio," in Proc. IEEE PIMRC, Athens, Greece, Sep. 2007

[25] Y. Zeng and Y. C. Liang, "Covariance based signal detections for cognitive radio," in Proc IEEE DYSPAN, Dublin, Ireland, Apr. 2007, pp. 202-207.

[26] Y. Zeng, C. L. Koh, and Y. C. Liang, "Maximum eigenvalue detection: Theory and application," in Proc. IEEE ICC, Beijing, China, May 2008, pp. 4160-4164.

[27] Z. Tian and G. B. Giannakis, "A wavelet approach to wideband spectrum sensing for cognitive radios," in Proc. IEEE CROWNCOM, Mykonos Island, Greece, Jun. 2006.

[28] S. M. Kay, Modern Spectral Estimation: Theory and Application. Englewood Cliffs, NJ: Prentice-Hall, 1988.
[29] Z. Cvetkovic and M. Vetterli, "Discrete-time wavelet extreme representation: Design and consistent reconstruction," IEEE Trans. Signal Process., vol. 43, pp. 681-693, Mar. 1995.

[30] S. Mallat and W. Hwang, "Singularity detection and processing with wavelets," IEEE Trans. Inf. Theory, pp. 617-643, Mar. 1992.

[31] J. Ma and Y. G. Li, "A probability-based spectrum sensing scheme for cognitive radio," in Proc. IEEE ICC, Beijing, China, May 2008, pp. 3416-3420.

[32] X. W. Zhou, Y. G. Li, Y. H. Kwon, and A. Soong, "Detection timing and channel selection for periodic spectrum sensing in cognitive radio," in IEEE Globecom'08, New Orleans, LA, Nov. 2008.

[33] G. Ganesan, Y. G. Li, B. Bing, and S. Li, "Spatiotemporal sensing in cognitive radio networks," IEEE J. Sel. Areas Commun., vol. 26, pp. 5-12, Jan. 2008.

[34] A. Ghasemi and E. S. Sousa, "Collaborative spectrum sensing for oppotunistic access in fading environments," in Proc. IEEE DYSPAN, Baltimore, MD, Nov. 2005, pp. 131-136.

[35] S. M. Mishra, A. Sahai, and R. W. Brodersen, "Cooperative sensing among cognitive radios," in Proc. IEEE ICC, Istanbul, Turkey, Jun. 2006, vol. 4, pp. 1658-1663.

[36] G. Ganesan and Y. G. Li, "Cooperative spectrum sensing in cognitive radio-Part I: Two user networks," IEEE Trans. Wireless Commun., vol. 6, pp. 2204-2213, Jun. 2007.

[37] G. Ganesan and Y. G. Li, “Cooperative spectrum sensing in cognitive radio-Part II Multiuser networks," IEEE Trans. Wireles Commun., vol. 6, pp. 2214-2222, Jun. 2007.

[38] J. Ma, G. D. Zhao, and Y. G. Li, "Soft combination and detection for cooperative spectrum sensing in cognitive radio networks," IEEE Trans. Wireles Commun., vol. 7, pp. 4502-4507, Nov. 2008.

[39] P. K. Varshney, Distributed Detection and Data Fusion. New York: Springer-Verlag, 1997.

[40] A. Ghasemi and E. S. Sousa, "Opportunistic spectrum access in fading channels through collaborative sensing," J. Commun., vol. 2, no. 2, pp. 71-82, Mar. 2007.

[41] J. N. Laneman and D. N. C. Tse, "Cooperative diversity in wireless networks: Efficient protocols and outage behaviour," IEEE Trans. Inf. Theory, vol. 50, pp. 3062-3080, Dec. 2004.

[42] J. N. Laneman and G. W. Wornell, "Distributed space-time coded protocols for exploiting cooperative diversity in wireless networks," IEEE Trans. Inf. Theory, vol. 49, pp. 2415-2425, Oct. 2003

[43] B. W. Kroeger, "Adjacent channel interference mitigation for FM digital audio broadcasting receivers," U.S. Patent 7221 917, May 22, 2007, issued.

[44] H. Yamaguchi, "Active interference cancellation technique for MB-OFDM cognitive radio," in Proc. 34th Eur. Microw. Conf., Amsterdam, The Netherlands, Oct. 2004, vol. 2, pp. 1105-1108.

[45] T. A. Weiss and F. K. Jondral, "Spectrum pooling: An innovative strategy for the enhancement of spectrum efficiency," IEEE Commun. Mag., Radio Commun. Suppl. pp. 8-14, Mar. 2004

[46] F. K. Jondral, "Software defined-radio-Basics and evolution to cognitive radio," EURASIP J.
Wireless Commun. Netw., no. 3, pp. 275-283, 2005.

[47] J. G. Proakis, Digital Communications, 3rd ed. New York: McGraw Hill, 1995.

[48] T. Weiss, J. Hillenbrand, A. Krohn, and F. K. Jondral, "Mutual interference in OFDM-based spectrum pooling systems," in Proc. 59th IEEE Veh. Tech. Conf., Milan, Italy, May 2004, vol. 4 pp. 1873-1877.

[49] S. Brandes, I. Cosovic, and M. Schnell, "Reduction of out-of-band radiation in OFDM systems by insertion of cancellation carriers," IEEE Commun. Lett., vol. 10, pp. 420-422, Jun. 2006.

[50] I. Cosovic, S. Brandes, and M. Schnell, "Subcarrier weighting: A method for sidelobe suppresion in OFDM systems," IEEE Commun. Lett., vol. 10, pp. 444-446, Jun. 2006.

[51] A. Oppenheim and R. Schafer, Digital Signal Processing. Englewood Cliffs, NJ Prentice-Hall, 1975.

[52] R. Rajbanshi, A. M. Wyglinski, and G. J. Minden, "An efficient implementation of NC-OFDM transceivers for cognitive radios," in Proc. IEEE CROWNCOM, Mykonos Island, Greece, Jun. 2006.

[53] J. D. Poston, “Discontiguous OFDM considerations for dynamic spectrum access in idle TV channels," in Proc. IEEE DYSPAN, Baltimore, MD, Nov. 2005, pp. 607-610.

[54] R. Rajbanshi, A. M. Wyglinski, and G. J. Minden, "Adaptive-mode peak-to-average power ratio reduction algorithm for OFDM-based cognitive radios,' in Proc. IEEE Veh. Technol. Conf., Montréal, PQ, Canada, Sep. 2006

[55] E. H. German, "Transform domain signal processing study final report," Reisterstown MD, Aug. 1988, Air Force F30602-86-C-0133, DTIC: ADB132635.

[56] V. D. Chakravarthy, A. K. Shaw, M. A. Temple, and J. P. Stephens, "Cognitive radio-An adaptive waveform with spectral sharing capability," in Proc. IEEE WCNC, New Orleans, LA, Mar. 2005, vol. 2, pp. 724-729.

[57] J. P. S. A. K. S. V. Chakravarthy, A. S. Nunez and M. A. Temple, "TDCS, OFDM, and MC-CDMA: A brief tutorial," IEEE Commun. Mag., vol. 43, pp. 11-16, Sep. 2005

[58] C. Han, J. Wang, S. Gong, and S. Li, "Performance of the OFDM-based transform domain communication system in cognitive radio contexts," in Proc. IEEE PIMRC, Helsinki, Finland, Sep. 2006

[59] Q. Zhao, L. Tong, and A. Swami, "Decentralized cognitive MAC for dynamic spectrum access," in Proc. IEEE DYSPAN, Baltimore, MD, Nov. 2005, pp. 224-232.

[60] X. Liu and W. Wang, "On the characterstics of spectrum-agile communication networks," in Proc. IEEE DYSPAN, Baltimore, MD, Nov. 2005, pp. 214-223.

[61] J. C. Dunat, D. Grandblaise, and C. Bonnet, "Efficient OFDMA distributed optimization algorithm exploiting multiuser diversity," in Proc. IEEE DYSPAN, Baltimore, MD, Nov. 2005, pp. 233-242.

[62] O. Ileri, D. Samardzija, and N. B. Mandyam, "Demand responsive pricing and competitive spectrum allocation via a spectrum server," in Proc. IEEE DYSPAN, Baltimore, MD, Nov. 2005, pp. 194-202. 


\section{ABOUT THE AUTHORS}

Jun Ma received the B.S. and M.S. degrees from the Department of Electronic Engineering and Information Science, University of Science and Technology of China, Hefei, in 2003 and 2006, respectively. He is currently pursuing the Ph.D. degree in the School of Electrical and Computer Engineering, Georgia Institute of Technology, Atlanta.

$\mathrm{His}$ research interests include cognitive radio and cooperative communication.

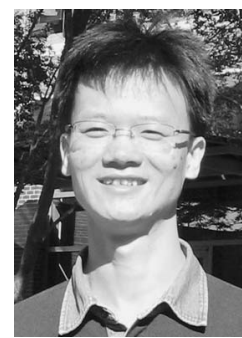

Geoffrey Ye Li (Fellow, IEEE) received the B.S.E. and M.S.E. degrees from the Department of Wireless Engineering, Nanjing Institute of Technology, Nanjing, China, in 1983 and 1986, respectively, and the Ph.D. degree from the Department of Electrical Engineering, Auburn University, Auburn, AL, in 1994.

He was a Teaching Assistant and then a Lecturer with Southeast University, Nanjing, China, from 1986 to 1991; a Research and Teaching Assistant

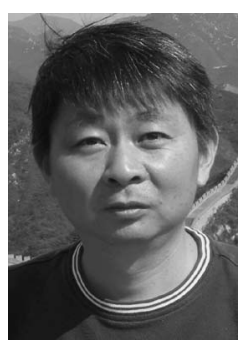
with Auburn University from 1991 to 1994; and a Postdoctoral Research Associate with the University of Maryland at College Park from 1994 to 1996. He was with AT\&T Labs-Research, Red Bank, NJ, as a Senior and then a Principal Technical Staff Member from 1996 to 2000. Since 2000, he has been with the School of Electrical and Computer Engineering, Georgia Institute of Technology, Atlanta, as an Associate and then a full Professor. $\mathrm{He}$ also has been a Visiting Chair Professor at the University of Electronic Science and Technology, China, since March 2006. His general research interests include statistical signal processing and telecommunications, with emphasis on OFDM and MIMO techniques, cross-layer optimization, and signal-processing issues in cognitive radios. In these areas, he has published about 200 papers in refereed journals or conferences and received about 20 patents. He is the author of (with Z. Ding) Blind Equalization and Identification (New York: Marcel Dekker, 2000) and (with G. Stüber) OFDM for Wireless Communications (New York: Springer, 2006). He is active in professional societies. He was or is an Editor, a member of the Editorial Board, or Guest Editor for six technical journals. He has organized and chaired many international conferences. Prof. Li was Technical Program Vice-Chair of the IEEE 2003 International Conference on Communications.
Biing Hwang (Fred) Juang (Fellow, IEEE) received the Ph.D. degree from the University of California, Santa Barbara.

He was with the Speech Communications Research Laboratory (SCRL) and Signal Technology, Inc. (STI) working on a number of government-sponsored research projects. His notable accomplishments during this period include development of vector quantization for voice applications, voice coders at extremely low bit rates,

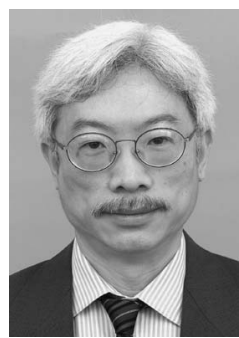
800 bps and around 300 bps, and robust vocoders for use in satellite communications. He was also a Co-Principal Investigator for the project on cochannel separation of speech signals sponsored by the U.S. government. He subsequently joined Bell Laboratories in 1982, working in the area of speech enhancement, coding, and recognition. He later became Director of Acoustics and Speech Research with Bell Labs and, at the turn of the century, Director of Multimedia Technologies Research with Avaya Labs (a spinoff of Bell Labs). His group continued the long heritage of Bell Labs in speech communication research. He has published extensively, including (with L. R. Rabiner) Fundamentals of Speech Recognition and has received about 20 patents. He joined the Georgia Institute of Technology, Atlanta, in 2002, where he holds the Motorola Foundation Chair Professorship and is an Eminent Scholar of the Georgia Research Alliance.

Prof. Juang is a Fellow of Bell Laboratories, a member of the U.S. National Academy of Engineering, and an Academician of Academia Sinica. He was Editor-in-Chief of the IEEE TRANSACTIONS ON SPEECH AND AUdio Processing and has held a number of positions in the IEEE Signal Processing Society, including Chair of its Fellow Evaluation Committee. He has received a number of technical awards, including several Best Paper awards in the area of speech communications and processing, the Technical Achievement Award from the IEEE Signal Processing Society, and the IEEE Third Millennium Medal. 